\title{
The rationale for the DREaM: Developing Research Excellence and Methods Project
}

\author{
Hazel Hall, Stephanie Kenna, Charles Oppenheim
}

\begin{abstract}
The article describes the background to the development of the DREaM project, which is aimed at expanding the range of skills of UK-based researchers in the LIS field, and at developing a network of active researchers, both in academia and amongst LIS practitioners. The project, which is funded by the Arts and Humanities Research Council involves two major conferences and a number of workshops throughout the UK starting in July 2011. Details of the events, and how the project will be evaluated, are provided.
\end{abstract}

\section{Introduction}

In November 2010 the DREaM project: Developing Research Excellence and Methods (Library and Information Science Research Coalition, 2011a) was awarded funds by the Arts and Humanities Research Council. The grant is held at Edinburgh Napier University and the project is supported by the Library and Information Science Research Coalition (http://lisresearch.org). The purpose of the grant is to develop a formal UK-wide network of Library and Information Science (LIS) researchers. The project started in January 2011 and runs until August 2012. The project is based upon five events in various UK locations at

\section{Authors}

Professor Hazel Hall is the Director of the Centre for Social Informatics at Edinburgh Napier University. She is the Principal Investigator of the DREaM project.

Stephanie Kenna works part-time for the Library and Information Science Research Coalition, having been involved in its development since 2006. She formerly held various positions at the British Library, including that of Research Analyst in the Research and Development Department. She provides support for the DREaM project.

Charles Oppenheim is former Professor of Information Science, and Head of Department of Information Science, Loughborough University

Email: c.oppenheim@btinternet.com 
which LIS researchers, and practitioners keen to undertake research will meet, exchange ideas and learn about methods with which they may not be familiar. The five DREaM project events will explore the scope of LIS and related research, and the range of methods appropriate to research in the domain. Researchers from LIS, as well as other subject domains, expert in a variety of techniques will present method overviews, covering their strengths and weaknesses. A wide range of techniques will be evaluated beyond the standard qualitative and quantitative methods commonly deployed in LIS research. It is hoped that participants from across all LIS sectors, and at different stages of their careers, will attend some or all of the events.

The first DREaM project event was a launch conference that took place in July 2011. Other events will take place between October 2011 and July 2012 in Edinburgh and London. This article describes the rationale for the project and what it hopes to achieve.

\section{The aims and objectives of DREaM}

The LIS profession in the UK is facing severe pressures. Many employers, both in the private and public sector, are rightly demanding justification for the expenditure of scarce resources on library and information services. The sector needs to respond to the challenge set by employers and paymasters by providing clear evidence of the value that the services provide to patrons, and to society at large. That evidence needs to be based on robust research leading to results that can be defended if challenged. That, in turn, requires a new approach by LIS professionals, who tend to rely on questionnaires and interviews, together with visit and issue statistics, to justify their services. In the latter case, such statistics are often collected because paymasters ask for them, not because librarians think that such statistics are a credible method of measuring value of service delivery. However, the need to use rigorous established research methods is not confined to those who need to justify their continued jobs and services provided. Many LIS professionals, both academics in LIS academic departments and practitioners elsewhere, have a genuine interest in undertaking more fundamental research to identify the principles underlying the profession and its practices.

From the outset a key assumption for the DREaM project was that the knowledge and experience of using non-traditional (to the LIS profession) yet wellestablished social sciences qualitative and quantitative research methods was limited amongst librarians and information scientists. Aligned to this was the desire to assist in developing skills in such methods. A second assumption was that LIS researchers often work in isolation, and may feel a lack of support from their managers, fellow professionals in their workplace, so the development of a network of those active in LIS research created by meeting at workshops and conferences would help them. Thus, the central theme of the project is that there are concerns about:

1. the quality and standing of LIS research in the UK;

2. the limited range of research methodologies and techniques deployed in LIS research; 
3. the lack of imagination displayed by LIS researchers and their reluctance to interact effectively with researchers in other fields;

4. the failure of current research endeavours to feed into practice, policy development and inform future research strategy and investment;

5. the lack of an evidence base on which the value and impact of library and information services can be assessed.

In addition, the research community in LIS is somewhat fragmented, so opportunities for new knowledge creation at the boundaries of the professional groupings are few.

The main aims of the DREaM project are:

1. To bring LIS researchers from range of backgrounds together in a new sustainable network with researchers from related disciplines to share and explore good practice and experience regarding appropriate methodologies and techniques for undertaking LIS research;

2. To address current challenges facing LIS research in the UK related to:

- Building capacity and capability in the development and implementation of innovative methodologies/techniques in undertaking LIS research;

- Raising the quality and standards of research training and practice in LIS;

- establishing a foundation for long-term research collaborations across LIS and related sectors;

- enhancing the value and impact of LIS research output with particular reference to policy development, improved services provision, and the informing of future LIS research agendas for investment.

Within these aims, key objectives are:

1. To develop a formal UK-wide network of LIS researchers to help spread expertise into a connected practitioner community across all LIS sectors through high quality training and opportunities for knowledge exchange;

2. To support researchers and practitioners in identifying and deploying the best research methods;

3. To raise understanding amongst researchers and practitioners of research methods new to them;

4. To develop strategies to meet the challenges facing the LIS research community.

\section{The potential benefits of the project}

There are three categories of potential beneficiaries of the project. The first comprises teaching and research staff who undertake LIS research. From a broad perspective, engagement in this programme will give these staff the opportunity both to contribute to the improvement in quality and standing of LIS research in the UK, and to benefit from the work of others. In the long-term they will undertake relevant, high priority research that can inform policy, determine 
information services provision, and develop the future research agenda in LIS. More specifically, this project allows LIS academics to invest in their own training and development by participating in the DREaM project events and/or engaging with the online resources generated by the project. Interactions with academic peers and - arguably more importantly - with practitioner researchers will enhance the possibilities of exploring avenues for new research initiatives as new knowledge is generated at the boundaries of professional groups. Fresh ideas and exposure to innovative research approaches will invigorate their working environment. The involvement of high profile speakers and facilitators from abroad will also provide individual LIS academics with a route to contacts beyond the UK. For LIS academics involved in teaching, this project will provide access to resources that can be repurposed for the classroom, such as case study material derived first-hand. Thus the project will improve teaching-research linkages, as well as research per se.

Other research-active academics in related subject domains (such as archives, museum studies, media and communication studies) may benefit from knowledge of the DREaM project's successes (and challenges), and the outputs that it produces. They will be able to access the project web site and learn about how the network is established and managed, as well as examine the resources for relevance to their own subject domains. DREaM may provide a blueprint for similar networks to be established in other subject areas.

The next key stakeholder group who will benefit are those LIS practitioners who are currently undertaking research, or wish to do so in the future. Some of these may have learned the basics of research techniques when they were at library school, but others may not have received such instruction, or have forgotten what they were taught years ago. Many will lack confidence in undertaking research for these reasons, or may wish to extend their range of skills to other methods.

Finally, library users across ALL disciplines will benefit indirectly from the project as end-users of services that are developed on the basis of robust research.

Specific provision has been made in the DREaM project budget for those at the start of their careers. For example, places for doctoral students at the launch conference in July 2011 were offered at a reduced registration rate and provision was made for a numbers of LIS professionals at an early stage of their career to attend, thanks to the sponsorship of the major LIS recruitment agents Glen Recruitment, Sue Hill Recruitment and TFPL.

The DREaM project will impact two main sets of non-academic beneficiaries. The most obvious of these, and the one where it will be first felt, is the extant community of LIS practitioner researchers. The number of beneficiaries in this category will grow as new researchers are recruited to the community as direct a result of awareness of, and engagement in, the programme. This may be achieved through active participation at DREaM events, or remotely as individuals are routed to the web-based resources produced as part of the programme.

Assessing the success of fostering practitioner interest in research and supporting their endeavours may be achieved by monitoring, for example: (1) increases in applications for research funding by practitioner researchers, (2) levels of 
practitioner success in winning resources to support their research; (3) the extent to which a fuller range of research methods is exploited in LIS research projects; (4) changes in the proportion of research literature in LIS published by practitioner (as opposed to academic) authors; and (5) the development of innovative new research programmes that are highly relevant to the UK research agenda.

Ultimately end-users of library and information services across all sectors public, commercial private and third - will benefit indirectly from practitioner researcher engagement in the DREaM project. At a local level, end-users will experience enhanced library and information services provision as a result of improvements in the quality and standard of LIS research conducted by their LIS staff. Equally, end-users will feel the positive long-term influence of research output of excellent quality on policy decisions, e.g., of the funding for LIS service provision. This is because a stronger collective "voice", which can draw on soundly completed LIS research output. Wider dissemination of new research output by practitioner and academic LIS researchers alike will also inform the future LIS research agendas, and this will help target investment towards identifiable research priorities, again with the ultimate goal of improvement in services offered to end-users. It is also possible to argue that the outcomes of this project may address wider economic and societal concerns of the population at large, not least from a public services perspective. This is on the basis that much work in public libraries is related to the quality of life of all citizens, regardless of their age, ethnic origin, level of educational attainment, job status etc.

An indirect outcome of the DREaM project may therefore be a contribution to the regeneration of public libraries as services provision improves due to decisions taken on the basis of a sound evidence base assembled by skilled researchers. Thus it might be argued that outputs from the project could contribute less obvious, though important, benefits to the local environments in which we work and live.

\section{The programme of events}

The first, event, held in July 2011, comprised a full programme of international speakers and had as its theme "out of the comfort zone". Full details can be found at http://lisresearch.org/dream-project/dream-event-1-launch-conference-tuesday$\underline{19-j u l y-2011 /}$

The next three one-day events take place in October 2011 in Edinburgh, January 2012 in London and April 2012 in Edinburgh. Each event will include presentations on basic philosophical approaches to research methodology, on quantitative methods, on qualitative methods, on other methods, and will discuss cross-cutting matters such as getting published in novel ways, legal and ethical issues, and how research affects policy. Each event will also include interactive sessions using games or exercises, Each event will cover different topics, so ideally attendees should attend all of them to get comprehensive coverage.

Each event follows a similar broad structure, and together, the three events cover most of the major methods identified as being worthy of consideration by LIS researchers by the project advisory board, together with issues associated with 
research, which apply to all the methods used. The content of the final conference in July 2012 has not yet been finalised. Readers are advised to check the project website for further details of these events as they are finalised.

\section{Dissemination activities for the project}

To ensure the project and its outcomes has as wide an impact as possible, arrangements have been made to use multiple channels for both the coordination of activities and the dissemination of results. The project itself has an online presence. Ultimately this will offer more than a standard repository for project material. For example, it will include space to foster collaboration online, and material recorded live at each of the DREaM events. More traditional project outputs such as this, and other journal articles and conference papers will be employed.

\section{Evaluation of the project}

Towards the end of the DREAM programme its impact will be assessed. One way will by use of a survey of attendees at the project's events (despite the comments above, the project team recognise the value of surveys!) to assess the impact of the project on their research skills, their predictions on how it may have changed the UK LIS research landscape, their actual use of resources produced by the project (both for research and teaching purposes), any partnerships that have emanated from involvement in the programme, and their opinions of the future direction of the domain and its leadership. It is not so easy to assess impact on the wider population. Even so, the project team will be alert to any changes or improvements that can be attributable to the DREaM project as far as general academic library provision is concerned.

It will be possible to measure some of the project outcomes within the time frame of the project itself. For example, it will be relatively straightforward to assess the perceptions of the extent of growth in research skills amongst practitioner researcher participants at DREaM events. The value of other outcomes will become evident at a later date. For example, if the project is successful in fostering a research culture amongst LIS practitioners, it is reasonable to expect to see a larger proportion of journal submissions by this constituency. However, that is for the longer term.

\section{References}

Library and Information Science Research Coalition (2011a) DREaM Project. URL: http://lisresearch.org/dream-project/ [accessed 10.09.11].

Library and Information Science Research Coalition (2011b) DREaM event 1 launch conference, Tuesday 19 July 2011, URL: http://lisresearch.org/dreamproject/dream-event-1-launch-conference-tuesday-19-july-2011/ [accessed 10.09.11]. 


\section{Acknowledgements}

We wish to thank the Arts and Humanities Research Council for funding the project. We also wish to thank the DREaM Project Advisory Board for their invaluable advice and guidance.

\section{Open access and copyright}

Library and Information Research is an open access journal. A freely available copy of this paper may be downloaded from the journal's website: http://www.cilipjournals.org.uk/lir

Copyright and associated moral rights in works published in Library and Information Research are retained by the author(s) but this paper may be used freely, with proper attribution, in educational and other non-commercial settings. 